\title{
State anxiety and information processing: A 7.5\% carbon dioxide challenge study
}

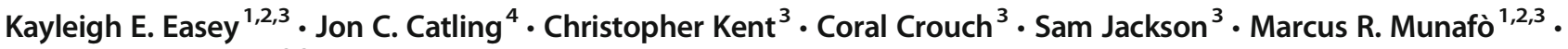 \\ Angela S. Attwood ${ }^{1,2,3}$
}

Published online: 1 February 2018

(C) The Author(s) 2018. This article is an open access publication

\begin{abstract}
We used the 7.5\% carbon dioxide model of anxiety induction to investigate the effects of state anxiety on simple information processing. In both high- and low-anxious states, participants $(n=36)$ completed an auditory-visual matching task and a visual binary categorization task. The stimuli were either degraded or clear, so as to investigate whether the effects of anxiety are greater when signal clarity is compromised. Accuracy in the matching task was lower during $\mathrm{CO}_{2}$ inhalation and for degraded stimuli. In the categorization task, response times and indecision (measured using mouse trajectories) were greater during $\mathrm{CO}_{2}$ inhalation and for degraded stimuli. For most measures, we found no evidence of Gas $\times$ Clarity interactions. These data indicate that state anxiety negatively impacts simple information processing and do not support claims that anxiety may benefit performance in low-cognitively-demanding tasks. These findings have important implications for understanding the impact of state anxiety in real-world situations.
\end{abstract}

Keywords Anxiety $\cdot$ Visual perception $\cdot$ Auditory perception $\cdot$ Human factors

Anxiety is a common feature of everyday life. From an evolutionary perspective, state anxiety may have adaptive value because it can focus attention to salient stimuli, particularly those that signal threat. However, "fight or flight" reactions are often inappropriate in modern situations, which require controlled and reasoned cognitive processing. For example, in many professions, including medicine, aviation, and the military, workers are required to process information, make reasoned decisions, and perform intricate skills in states of heightened anxiety. Impairments in any of these processes can have disastrous consequences. Indeed, it is estimated that the majority of fatal aviation accidents can be linked to human

Angela S. Attwood

angela.attwood@bristol.ac.uk

1 MRC Integrative Epidemiology UnitUniversity of Bristol, Bristol, UK

2 UK Centre for Tobacco and Alcohol Studies, University of Bristol, Bristol, UK

3 School of Experimental Psychology, University of Bristol, 12a Priory Road, Bristol BS8 1TU, UK

4 School of Psychology, University of Birmingham, Birmingham, UK error (International Air Transport Association, 2013). Understanding how anxiety affects these abilities, and how we may attenuate impairments, is an important area of research with wide-reaching implications.

High trait anxiety has been associated with poorer outcomes on a number of cognitive parameters, in both animals (Herrero, Sandi, \& Venero, 2006) and humans (Byron \& Khazanchi, 2011; Gawda \& Szepietowska, 2016). Traditionally, anxiety has been associated with a hypersensitivity to threat (Spielberger, 1972). However, more recently, Bishop (2009) suggested that a broader dysregulation of the attentional control system underlies more generic impairments in cognitive performance in high trait anxiety.

There is increasing evidence that state anxiety may have similar negative effects on cognition. State anxiety is a fundamental component of the anxiety profile, since its frequency and severity are heightened in trait-anxious individuals. Furthermore, anxious states are not restricted to "high trait" subgroups. In numerous situations, individuals have to perform in anxiogenic conditions, even though skills are routinely trained and practiced in nonanxious states. Because these situations often involve complex tasks, the extent to which anxiety negatively impacts on different cognitive processes is often unclear. It is plausible that under some circumstances, 
heightened state anxiety might benefit performance, particularly when cognitive load is low (Diaper et al., 2012).

In the present study, we investigated the effects of state anxiety on simple auditory and visual information processing. Because real-world telecommunication devices are prone to interference that degrades signal quality, and visual stimuli may be viewed in suboptimal conditions (e.g., blurred CCTV), we included both clear and degraded stimuli in both tasks. We used the 20-min 7.5\% $\mathrm{CO}_{2}$ inhalation challenge (Bailey, Argyropoulos, Kendrick, \& Nutt, 2005) to induce state anxiety. This model has produced reliable increases in state anxiety in a number of studies (Attwood, Catling, Kwong, \& Munafò, 2015; Attwood et al., 2017; Attwood, Penton-Voak, Burton, \& Munafò, 2013; Button, Lewis, Penton-Voak, \& Munafò, 2013; Garner, Attwood, Baldwin, James, \& Munafò, 2011), and although there is evidence that its effects might be somewhat larger in individuals prone to anxiety (Fluharty, Attwood, \& Munafò, 2016), in our experience nonresponders are rare. We hypothesized that performance would be poorer during $7.5 \% \mathrm{CO}_{2}$ inhalation (as compared to inhalation of air) and in the degraded (as compared to the clear) condition, and that the magnitude of the degradation effect would be greater for $\mathrm{CO}_{2}$ than for air inhalation.

\section{Method}

The protocol for this study was preregistered on the Open Science Framework: https://osf.io/gqs93/. Ethics approval was obtained from the Faculty of Science Research Ethics Committee at the University of Bristol (reference: 30101413461).

\section{Participants}

Thirty-six healthy volunteers (23 female, 13 male) were recruited from among members of the University of Bristol and the local community. The exclusion criteria included being under 18 or over 50 years of age, daily smoking, history of drug/alcohol dependency, pregnancy or breast feeding, recent use of prescribed or illicit drugs, uncorrected visual or hearing problems, diagnosed medical illness, and not being registered with a general practitioner. Pregnancy and recent drug use were assessed by urine screen, whereas all other criteria were confirmed by self-report. Participants were also excluded if they had high systolic or diastolic blood pressure (SBP/ DBP) $(<140 / 90 \mathrm{mmHg})$, bradycardia or tachycardia $(<50$ or $>90$ beats per minute), or a body mass index (BMI) outside a healthy range $\left(<18\right.$ or $>30 \mathrm{~kg} / \mathrm{m}^{2}$ ) (all physically assessed). Psychiatric health was assessed using a truncated MINIInternational Neuropsychiatric Interview (Sheehan et al., 1998). Participants refrained from consuming alcohol for $36 \mathrm{~h}$ prior to the study day. Expired breath alcohol and carbon monoxide readings were taken, and participants were excluded if the readings were $>0$ or $\geq 10$, respectively. To achieve our target of 36 participants, a total of 45 individuals were screened. The nine participants who were not enrolled were deemed ineligible because they had either failed to meet one or more of the eligibility criteria or did not respond to further contact.

The sample size was determined from the effect sizes obtained in a previous study investigating the effects of $\mathrm{CO}_{2}$ inhalation on speech perception (Mattys, Seymour, Attwood, $\&$ Munafò, 2013). This study indicated an effect size of $d z=$ 0.57 (difference in phoneme recognition during $7.5 \% \mathrm{CO}_{2}$ inhalation vs. air inhalation). On the basis of these data, we required a sample size of $n=36$ to achieve $90 \%$ power at an alpha level of $5 \%$.

\section{Design}

Both gas (air, 7.5\% $\mathrm{CO}_{2}$ ) and stimulus clarity (clear, degraded) were manipulated within subjects. The gas and task orders were counterbalanced across participants.

\section{Materials}

The gases were either $7.5 \% \mathrm{CO}_{2} / 21 \%$ oxygen $/ 71.5 \%$ nitrogen or medical air ( $21 \%$ oxygen; BOC Ltd.). These were administered using an oro-nasal mask (Hans Rudolph, Kansas City, MO, USA). For safety reasons, the gas was administered single blind. Questionnaires ${ }^{1}$ included the Spielberger State-Trait Anxiety Inventory (state and trait; Spielberger, Gorsuch, Lushene, Vagg, \& Jacobs, 1983), Positive and Negative Affect Schedule (PANAS; Watson, Clark, \& Tellegen, 1988), Anxiety Sensitivity Index (ASI) (Derryberry \& Reed, 2002), and Eysenck Personality Questionnaire Revised (EPQR-R) (Eysenck \& Eysenck, 1991).

The computer tasks were displayed on a $17-$ in. LCD monitor at a resolution of $1,280 \times 1,024$ with a $60-\mathrm{Hz}$ refresh rate. Each task was completed twice (once during each inhalation).

\section{Audio-visual matching task}

On each trial, a black-and-white image was presented centrally on screen and a verbal descriptor was played simultaneously through headphones. On $50 \%$ of trials the image matched the verbal descriptor. Participants were required to indicate whether the descriptor matched the image by pressing designated keys on the keyboard. If a response was not made within $4,000 \mathrm{~ms}$, the trial was terminated. On $50 \%$ of the trials the descriptor was clear, and on $50 \%$ it was degraded (i.e., muffled). The audio clips were degraded using low-pass filtering

\footnotetext{
${ }^{1}$ Full details of the sample means and standard deviations are available upon request.
} 
with a $1,000-\mathrm{Hz}$ cutoff and a 32-dB/octave roll-off, resulting in them sounding muffled. Therefore, four conditions were presented (clear/matching, degraded/matching, clear/ nonmatching, degraded/nonmatching), each comprising 50 trials. The outcome variables were reaction time (in milliseconds; correct responses only), hit rate (i.e., number of correct responses in matching trials), and false alarm rate (i.e., number of errors made on nonmatched trials, in which participants reported that the visual and auditory stimuli matched when the stimuli did not).

\section{Visual binary categorization task}

A schematic of the task is shown in Fig. 1. On each trial, a pictorial stimulus appeared centrally on the screen. Participants were required to identify whether the image was biological or nonbiological. The labels "Bio" and "Non" appeared in response boxes in the top right and left of the screen (positions counterbalanced across participants). Participants responded using a standard USB mouse.

The stimuli consisted of 160 color $190 \times 190$ pixels $(5.72 \times$ $5.72 \mathrm{deg}$ of visual angle) images taken from the Bank of Standardized Stimuli (Brodeur, Dionne-Dostie, Montreuil, \& Lepage, 2010), 50\% of which were biological items and 50\% nonbiological items. Furthermore, $50 \%$ of the images in each category were degraded using a 50\% Gaussian blur. Category type (biological or nonbiological) and image type (clear or degraded) were randomly intermixed.

Each trial was initiated by clicking within a "Start" box located at the bottom of the screen. A randomly selected stimulus was then presented, and participants were instructed to respond as quickly and as accurately as possible. To encourage online decision making, a prompt appeared at end of any trial on which participants failed to move the mouse within $1,000 \mathrm{~ms}$ of stimulus onset. There was a $500-\mathrm{ms}$ intertrial interval, and the task consisted of 80 trials. The stimulus presentation and response collection were achieved via MouseTracker software (Freeman \& Ambady, 2010), which has been used in previous categorization studies (Dale, Kehoe, \& Spivey, 2007).

The outcome variables for this task comprised: (1) reaction time (correct responses), measured from the point at which the "Start" button was clicked to the point at which the participant clicked on either the "Bio" or "Non" response box (i.e., until the response was terminated), and (2) average area under the curve (AUC), which is a measure of attraction to the incorrect response and therefore gives an indication of indecision (with higher AUCs representing greater indecision; see panel D of

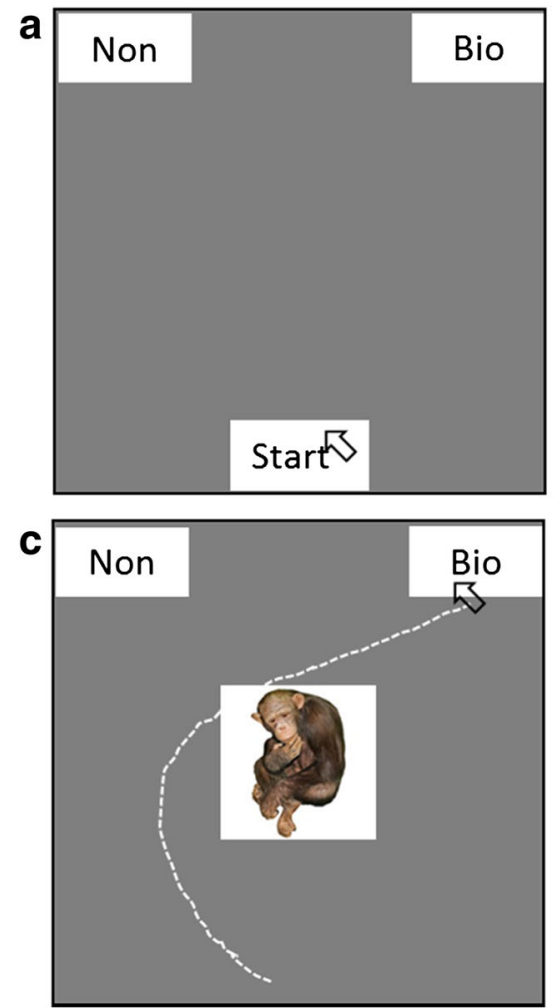

Fig. 1 Structure of a trial $(\mathrm{A}-\mathrm{C})$ for the binary categorization task. (A) Participants initiated the trial by clicking the "Start" button. (B) An image (clear, in the example) was displayed. (C) Participants moved the mouse cursor (broken white line, not visible on trial) and clicked on a response option. (D) Area under the curve (AUC; i.e., the filled area) is the area

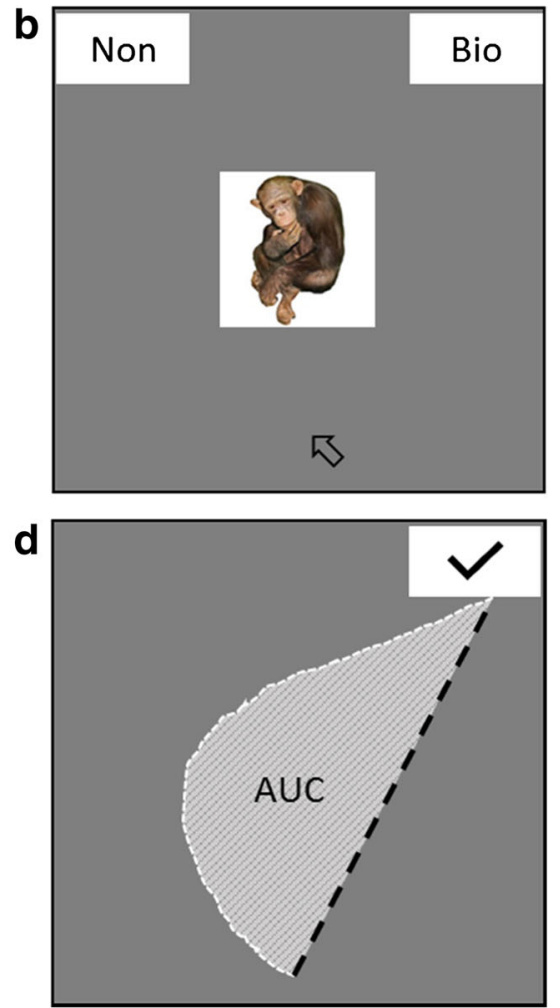

between the actual trajectory (broken white line) and an idealized trajectory (broken black line, drawn from the starting $x, y$ position to the finishing $x, y$ position of the actual trajectory). Note that the images are not to scale. 
Fig. 1). Before AUC was calculated, screen coordinates were transformed into a standard coordinate space from $(-1,1.5$; top left) to (1, 0; bottom right); all response trajectories to the left response were flipped onto the right response (allowing us to compare the biological and nonbiological images). We also time normalized all trajectories via linear interpolation into 100 time bins, to allow us to compare the spatial trajectories of responses with different reaction times (see Freeman \& Ambady, 2010, for fuller discussion of the analysis techniques).

\section{Procedure}

Prior to the session, a telephone screen assessed basic eligibility. Eligible participants attended a single test session, at which full written informed consent was obtained and further screening assessments were conducted. If eligibility was met, baseline questionnaire (STAI, SSAI, PANAS, and ASI) and cardiovascular (blood pressure [BP] and heart rate [HR]) measures were recorded. The inhalation began with $60 \mathrm{~s}$ of free breathing before the tasks were started (this allowed for the gas to start taking effect before data collection began). Inhalations then continued for the duration of the two computer tasks (up to $20 \mathrm{~min}$ for each inhalation). Immediately after each inhalation, measures of BP, HR, SSAI, and PANAS were completed, and there was a 30-min washout period between inhalations. The second inhalation followed the same procedure as the first. After the inhalations were complete, participants remained in the room for a minimum of $20 \mathrm{~min}$, to allow any effects to dissipate, and completed the EPQ-r. Participants were then debriefed and reimbursed. A followup call was conducted $24 \mathrm{~h}$ later to assess whether any adverse events had occurred.

\section{Results}

The data that form the basis of the results presented here are available from the University of Bristol Research Data
Repository (http://data.bris.ac.uk/data/), (https://doi.org/10. 5523/bris. 1bvk8fmcvv4m1b6c1p5efsgvr).

Task data were assessed for normality, and where deviations were observed, appropriate transformations were run. For ease of interpretation, untransformed data and analyses are presented where the results did not differ after transformation.

\section{Characteristics of participants}

The participants $(\mathrm{n}=36 ; 39 \%$ male) were between 19 and 49 years of age $(M=22, S D=5)$. Their body mass indexes ranged between 18 and $29(\mathrm{M}=23, \mathrm{SD}=3)$. In terms of test results, the participants' STAI Trait and ASI scores ranged between 22 and $46(\mathrm{M}=32, \mathrm{SD}=7)$ and between 2 and 31 $(\mathrm{M}=15, \mathrm{SD}=6)$, respectively. Their EPQ-R scores ranged between 0 and $13(\mathrm{M}=6, \mathrm{SD}=3)$ for psychoticism, 2 and 21 $(\mathrm{M}=9, \mathrm{SD}=4)$ for neuroticism, and 8 and $23(\mathrm{M}=17, \mathrm{SD}=$ 4) for extraversion.

\section{Subjective and cardiovascular effects}

PANAS-negative data in the air condition were positively skewed due to two outlying scores, which were likely due to high anticipatory anxiety in the air condition. Removing these data points increased the comparison effect size but did not change the overall effect, and therefore the data from the full sample are reported. State anxiety (STAI), negative affect (PANAS-negative), SBP, DBP, and HR were higher, and positive affect (PANAS-positive) was lower, after $\mathrm{CO}_{2}$ than after air inhalation (see Table 1), confirming the validity of the anxiety manipulation.

\section{Audio-visual task}

For accuracy, hit rate and false alarm rate were calculated separately for the clear and degraded conditions. The mean reaction time (in milliseconds) was calculated for correct responses (matching trials only). The total hit data were

Table 1 State anxiety, affect and cardiovascular $t$ test comparison data

\begin{tabular}{|c|c|c|c|c|c|c|c|}
\hline & $\begin{array}{l}\text { Mean Difference } \\
(S D) \text { : Air vs. } \mathrm{CO}_{2}\end{array}$ & Delta $\% \mathrm{CO}_{2}$ & $\begin{array}{l}\text { Delta \% } \\
\text { Air }\end{array}$ & $\begin{array}{l}\text { Effect } \\
\text { Size }(d z)\end{array}$ & $d f$ & $95 \% \mathrm{CI}$ & $p$ Value \\
\hline STAI State & $20.8(10.7)$ & 97.3 & 20.4 & 1.95 & 35 & 17.2 to 24.4 & $<.001$ \\
\hline PANAS-pos. & $-6.6(6.4)$ & -35.8 & -17.1 & 1.03 & 35 & -8.7 to -4.4 & $<.001$ \\
\hline PANAS-neg. & $9.9(7.1)$ & 97.7 & 7.8 & 1.40 & 35 & 7.5 to 12.3 & $<.001$ \\
\hline SBP & 13.4 (12.6) & 13.3 & 0.4 & 1.06 & 35 & 9.1 to 17.7 & $<.001$ \\
\hline DBP & $5.0(12.8)$ & 10.9 & 3.4 & 0.40 & 35 & 0.7 to 9.3 & .025 \\
\hline HR & $16.3(18.5)$ & 25.8 & 1.8 & 0.88 & 35 & 10.0 to 22.5 & $<.001$ \\
\hline
\end{tabular}

STAI, Spielberger State-Trait Anxiety Inventory; PANAS, Positive and Negative Affect Schedule; SBP, systolic blood pressure; DBP, diastolic blood pressure; HR, heart rate. Delta scores are derived from the group means. 


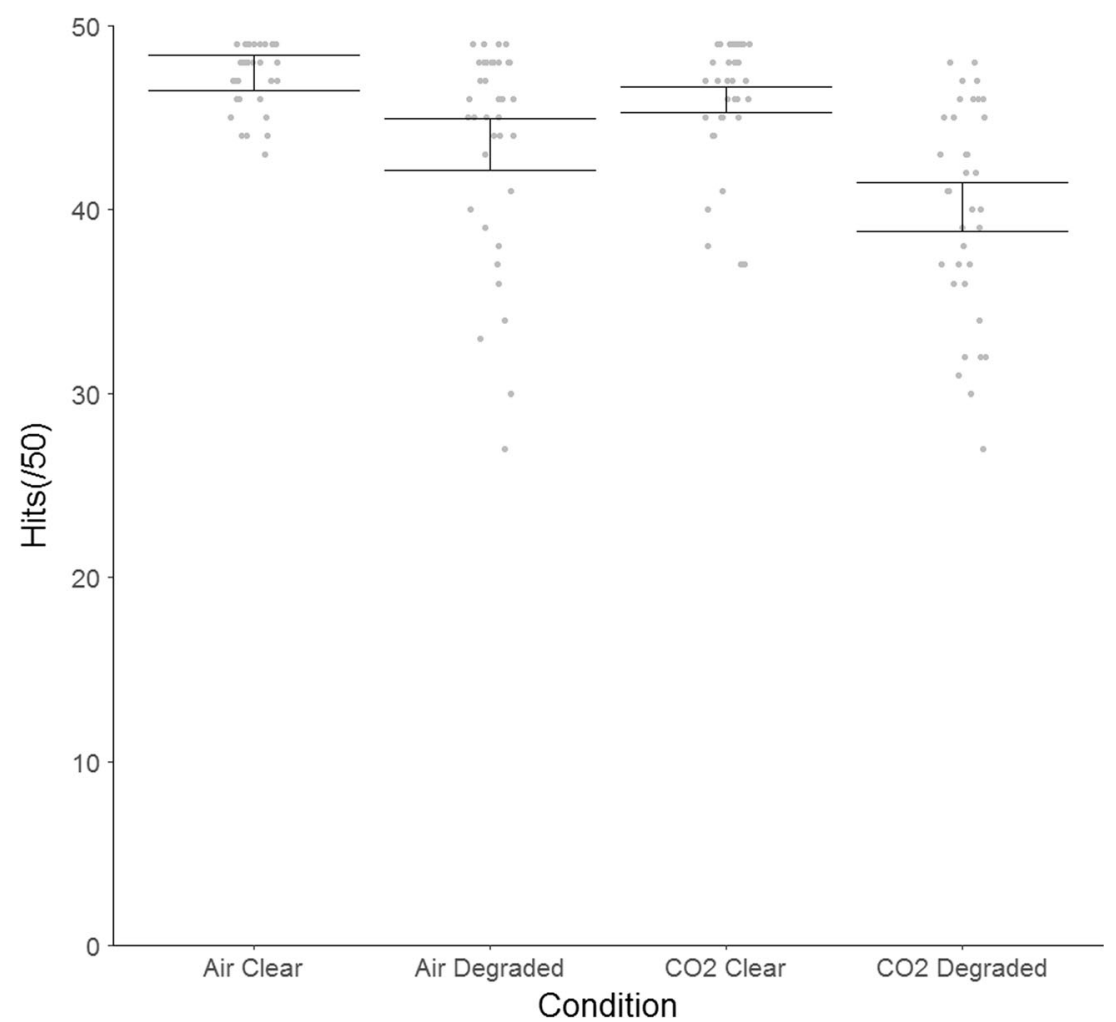

Fig. 2 Mean hits (out of 50) in the clear and degraded conditions during $\mathrm{CO}_{2}$ and air inhalation (untransformed data). Circles are the individual data points, and horizontal lines represent within-subjects confidence intervals, calculated using Morey (2008).

negatively skewed, and $\log _{10}$ transformations were used to correct this (Tabachnick \& Fidell, 2007).

Using untransformed data, we observed a main effect of gas on hit rates $\left[F(1,35)=18.50, p<.001, \eta_{\mathrm{p}}^{2}=.35\right]$, indicating more hits in the air than in the $\mathrm{CO}_{2}$ condition. We also found a main effect of clarity $\left[F(1,35)=69.42, p<.001, \eta_{\mathrm{p}}{ }^{2}=\right.$ $.67]$, indicating greater accuracy when the verbal descriptor was clear than when it was degraded (see Fig. 2). There was weak evidence of a Gas $\times$ Clarity interaction $[F(1,35)=3.32$, $\left.p=.077, \eta_{\mathrm{p}}{ }^{2}=.09\right]$ ]. Post-hoc $t$ tests stratified by gas revealed that although there were fewer hits in the degraded than in the clear condition during both air $(t=4.46, d f=35, p<.001, d z=$ $0.73,95 \%$ CI: 2.10 to 5.62$)$ and $\mathrm{CO}_{2}(t=8.27, d f=35, p=<$ $.001, d z=1.38,95 \%$ CI: 4.38 to 7.23$)$ inhalation, the magnitude of this effect was greater in the $\mathrm{CO}_{2}$ condition (as indicated by a greater effect size). This interaction was no longer observed once the data were transformed $[F(1,35)=0.19, p>$ $\left..250, \eta_{\mathrm{p}}^{2}=.005\right]$.

For false alarms, one outlier was observed and removed from the analysis. There was evidence of main effects of both gas $\left[F(1,34)=10.45, p=.003, \eta_{\mathrm{p}}{ }^{2}=.24\right]$ and clarity $[F(1,34)$ $\left.=15.05, p<.001, \eta_{\mathrm{p}}{ }^{2}=.31\right]$, with more false alarms during $\mathrm{CO}_{2}(M=2.2, S D=2.2)$ than during air $(M=1.1, S D=1.0)$ inhalation, and in the degraded $(M=2.1, S D=1.6)$ than in the clear $(M=1.2, S D=1.6)$ conditions. We found no evidence of a Gas $\times$ Clarity interaction $(p=.82)$.
There was evidence of a positive skew in mean reaction times. The findings did not differ using the transformed data, and therefore only statistics from the untransformed analysis are presented. Main effects of both gas $\left[F(1,35)=5.93, p=.020, \eta_{\mathrm{p}}{ }^{2}=.15\right]$ and clarity $[F(1$, $\left.35)=57.88, p<.001, \eta_{\mathrm{p}}{ }^{2}=.63\right]$ emerged, with slower reaction times during $\mathrm{CO}_{2}$ inhalation $(M=908 \mathrm{~ms} S D=$ $204 \mathrm{~ms})$ than during air inhalation $(M=840 \mathrm{~ms}, S D=171$ $\mathrm{ms})$, and in degraded $(M=916 \mathrm{~ms}, S D=170 \mathrm{~ms})$ than in clear $(M=833 \mathrm{~ms}, S D=173 \mathrm{~ms})$ conditions. There was no evidence of a Gas $\times$ Clarity interaction $(p>.250)$.

\section{Visual binary categorization task}

Only correct responses were analyzed (96\% of trials). Responses that were initiated after 1,000 ms were discarded ( $1 \%$ of trials).

Reaction time A $2 \mathrm{Gas}\left(\mathrm{CO}_{2}\right.$, air $) \times 2$ Clarity (clear, degraded) repeated measures analysis of variance (ANOVA) was conducted on correct reaction times. We found evidence of main effects of gas $\left[F(1,35)=6.43, p=.016, \eta_{\mathrm{p}}{ }^{2}=.16\right]$ and clarity $\left[F(1,35)=35.37, p<.001, \eta_{\mathrm{p}}{ }^{2}=.50\right]$. Reaction times were slower during $\mathrm{CO}_{2}(M=1,411 \mathrm{~ms}, S D=338 \mathrm{~ms})$ than during air $(M=1,279 \mathrm{~ms}, S D=202 \mathrm{~ms})$ inhalation, and for degraded ( $M=1,408 \mathrm{~ms}, S D=317 \mathrm{~ms})$ than for clear $(M=1,282 \mathrm{~ms}$, 
$S D=235 \mathrm{~ms}$ ) images. There was no clear evidence of a Gas $\times$ Clarity interaction $(p=.125)$, and no evidence of main effects or interactions for the average time to move the mouse $(F \mathrm{~s}<$ 0.34 ), suggesting that participants were trying to move the mouse as soon as the stimulus appeared across all conditions.

Area under the curve Density plots of the pooled AUC data did not show evidence of bimodality (Hartigan's $D=0.002, p$ $=.999)$. We therefore conducted a $2 \mathrm{Gas}\left(\mathrm{CO}_{2}\right.$, air $) \times 2$ Clarity (clear, degraded) repeated measures ANOVA on the average AUC values. The AUC values were higher after $\mathrm{CO}_{2}(M=$ $0.68, S D=0.44)$ than after $\operatorname{air}(M=0.57, S D=0.38)$ inhalation $\left[F(1,35)=15.03 p<.001, \eta_{\mathrm{p}}{ }^{2}=.30\right]$, suggesting greater indecision when anxious. The AUC values were also higher for the degraded images $(M=0.67, S D=0.42)$ than for the clear images $(M=0.58, S D=0.41)[F(1,35)=5.99 p=.020$, $\left.\eta_{\mathrm{p}}{ }^{2}=.15\right]$, suggesting that participants were more indecisive when the images were degraded. There was no evidence of an interaction between gas and clarity $(p=.97)$.

\section{Discussion}

We found evidence that state anxiety impairs information processing during simple cognitive tasks. These impairments consisted of fewer hits and more false alarms in the auditory-visual task and slower reaction times and greater indecision in the visual categorization task. These data support our hypothesis that performance suffers when participants are in an anxious state.

In contrast, the data did not support the hypothesis that the detrimental effect of stimulus degradation would be greater during $\mathrm{CO}_{2}$ inhalation. Other possible explanations of this lack of interaction between gas and stimulus degradation may be that the mechanism by which anxiety affects simple processing is different from the processes involved in differentiating clear and degraded stimuli. That is, the two main effects may have operated at different levels of cognition. For both tasks, performance was worse when the stimuli were degraded, indicating that the degradation manipulation was successful. However, it may be that the degree of difference in difficulty was not large enough to detect reliable differences between the gas inhalations, and effects of difficulty might be more apparent with more complex tasks. Observation of the means, particularly the hit data in the auditory-visual task, indicated that degradation effects were greater with $\mathrm{CO}_{2}$ but that this effect was not robust enough to be supported statistically. It is plausible that there was a small interaction effect that this study was not sufficiently powered to detect, and this warrants further investigation.
These findings are particularly noteworthy because the tasks required very simple information processing. Previous studies on attentional processing have indicated that the negative influence of anxiety may be limited to complex tasks, and that there may be some benefit of acute anxiety when tasks are simple (Diaper et al., 2012). Our data indicate that other cognitive processes, such as information processing and simple decision making, are negatively affected by state anxiety even when cognitive demand is low. It is plausible that processing advantages of state anxiety might arise when there is a need to process threat-related information. In support of this conjecture, Garner et al. (2011) reported difficulties in disengaging from threatening information during $7.5 \%$ $\mathrm{CO}_{2}$ inhalation. Although this was interpreted as impaired performance (since the aim of the task was to focus on nonthreat stimuli), this implies that there might be a benefit of anxiety if the aim of the task is to focus attention on threat information. However, our findings suggest that in situations in which this is not the primary aim, state anxiety negatively affects performance even in very simple tasks.

These findings have particular importance for realworld applications, where information processing and decision making are required in stressful conditions. For example, disruption to the processing of simple auditory information through communication systems such as twoway radios could have serious consequences for professionals involved in maintaining the safety of others (e.g., communication between pilots and air traffic control). This emphasizes a need for professional training paradigms to take account of these processing impairments, to mitigate their impact when skills have to be applied outside of the training contexts.

Future research could extend these findings by increasing task complexity in order to investigate whether gas-clarity interactions are more robust when the tasks are more cognitively demanding. The importance of this work is exemplified by the recent emergence of cognitive-training paradigms. The tractable nature of these processes mean that $\mathrm{CO}_{2}$ inhalation could be paired with training in order to improve anxietyrelated performance.

Author note A.S.A. and M.R.M. are members of the UK Centre for Tobacco and Alcohol Studies. Funding from the British Heart Foundation, Cancer Research UK, Economic and Social Research Council, Medical Research Council, and National Institute for Health Research, under the auspices of the UK Clinical Research Collaboration, is gratefully acknowledged. This study was supported in part by the Medical Research Council and the University of Bristol (Grant No. MC_UU_12013/6). A.S.A., J.C.C., and C.K. developed the study concepts. All authors contributed to the study design 
and protocol development. A.S.A., J.C.C., C.K., and S.J. developed the study materials (including the computer tasks and stimuli). The testing and data collection were conducted by S.J. and C.C. Finally, K.E.E., C.C., S.J., C.K., and A.S.A. performed the data analyses, and A.S.A. and K.E.E. drafted the manuscript. All authors contributed to interpretation of the results and critical review of the manuscript. All authors approved the final version of the manuscript for submission. A.S.A. and M.R.M. have acted as consultants for pharmaceutical companies on use of hypercapnic challenges in psychopharmacological research. The authors have no other conflicts of interest.

Open Access This article is distributed under the terms of the Creative Commons Attribution 4.0 International License (http:// creativecommons.org/licenses/by/4.0/), which permits unrestricted use, distribution, and reproduction in any medium, provided you give appropriate credit to the original author(s) and the source, provide a link to the Creative Commons license, and indicate if changes were made.

\section{References}

Attwood, A. S., Catling, J. C., Kwong, A. S., \& Munafò, M. R. (2015). Effects of $7.5 \%$ carbon dioxide $\left(\mathrm{CO}_{2}\right)$ inhalation and ethnicity on face memory. Physiology and Behaviour, 147, 97-101. https://doi. org/10.1016/j.physbeh.2015.04.027

Attwood, A. S., Easey, K. E., Dalili, M. N., Skinner, A. L., Woods, A., Crick, L.,. .. Munafò, M. R. (2017). State anxiety and emotional face recognition in healthy volunteers. Royal Society Open Science, 4, 160855. https://doi.org/10.1098/rsos.160855

Attwood, A. S., Penton-Voak, I. S., Burton, A. M., \& Munafò, M. R. (2013). Acute anxiety impairs accuracy in identifying photographed faces. Psychological Science, 24, 1591-1594. https://doi.org/10. 1177/0956797612474021

Bailey, J. E., Argyropoulos, S. V., Kendrick, A. H., \& Nutt, D. (2005). Behavioral and cardiovascular effects of $7.5 \% \mathrm{CO}_{2}$ in human volunteers. Depression and Anxiety, 21, 18-25.

Bishop, S. J. (2009). Trait anxiety and impoverished prefrontal control of attention. Nature Neuroscience, 12, 92-98. https://doi.org/10.1038/ nn. 2242

Brodeur, M. B., Dionne-Dostie, E., Montreuil, T., \& Lepage, M. (2010). The Bank of Standardized Stimuli (BOSS), a new set of 480 normative photos of objects to be used as visual stimuli in cognitive research. PLoS ONE, 5,e10773:1-13. https://doi.org/10.1371/journal. pone. 0010773

Button, K., Lewis, G., Penton-Voak, I., \& Munafò, M. (2013). Social anxiety is associated with general but not specific biases in emotion recognition. Psychiatry Research, 210, 199-207. https://doi.org/10. 1016/j.psychres.2013.06.005

Byron, K., \& Khazanchi, S. (2011). A meta-analytic investigation of the relationship of state and trait anxiety to performance on figural and verbal creative tasks. Personality and Social Psychology Bulletin, 37, 269-283. https://doi.org/10.1177/0146167210392788
Dale, R., Kehoe, C., \& Spivey, M. J. (2007). Graded motor responses in the time course of categorizing atypical exemplars. Memory \& Cognition, 35, 15-28.

Derryberry, D., \& Reed, M. A. (2002). Anxiety-related attentional biases and their regulation by attentional control. Journal of Abnormal Psychology, 111, 225-236.

Diaper, A., Nutt, D. J., Munafo, M. R., White, J. L., Farmer, E. W., \& Bailey, J. E. (2012). The effects of $7.5 \%$ carbon dioxide inhalation on task performance in healthy volunteers. Journal of Psychopharmacology, 26, 487-496. https://doi.org/10.1177/ 0269881111415729

Eysenck, H. J., \& Eysenck, S. B. G. (1991). Eysenck Personality Scales. London, UK: Hodder \& Stoughton.

Fluharty, M. E., Attwood, A. S., \& Munafò, M. R. (2016). Anxiety sensitivity and trait anxiety are associated with response to $7.5 \%$ carbon dioxide challenge. Journal of Psychopharmacology, 30, 182-187. https://doi.org/10.1177/0269881115615105

Freeman, J. B., \& Ambady, N. (2010). MouseTracker: Software for studying real-time mental processing using a computer mousetracking method. Behavior Research Methods, 42, 226-241. https://doi.org/10.3758/BRM.42.1.226

Garner, M., Attwood, A., Baldwin, D. S., James, A., \& Munafò, M. R. (2011). Inhalation of 7.5\% carbon dioxide increases threat processing in humans. Neuropsychopharmacology, 36, 1557-1562. https:// doi.org/10.1038/npp.2011.15

Gawda, B., \& Szepietowska, E. (2016). Trait anxiety modulates brain activity during performance of verbal fluency tasks. Frontiers in Behavioral Neuroscience, 10, 10. https://doi.org/10.3389/fnbeh. 2016.00010

Herrero, A. I., Sandi, C., \& Venero, C. (2006). Individual differences in anxiety trait are related to spatial learning abilities and hippocampal expression of mineralocorticoid receptors. Neurobiology of Learning and Memory, 86, 150-159. https://doi.org/10.1016/j.nlm. 2006.02.001

International Air Transport Association. (2013). IATA Safety Report. Retrieved from www.iata.org/publications/Documents/iata-safetyreport-2013.pdf

Mattys, S. L., Seymour, F., Attwood, A. S., \& Munafò, M. R. (2013). Effects of acute anxiety induction on speech perception: Are anxious listeners distracted listeners? Psychological Science, 24, 16061608. https://doi.org/10.1177/0956797612474323

Morey, R. D. (2008). Confidence intervals from normalized data: A correction to Cousineau (2005). Tutorial in Quantitative Methods for Psychology, 4, 61-64. https://doi.org/10.3758/s13414-012-0291-2

Sheehan, D. V., Lecrubier, Y., Sheehan, K. H., Amorim, P., Janavs, J., Weiller, E.,... Dunbar, G. C. (1998). The Mini-International Neuropsychiatric Interview (M.I.N.I.): The development and validation of a structured diagnostic psychiatric interview for DSM-IV and ICD-10. Journal of Clinical Psychiatry, 59, 22-33.

Spielberger, C. D. (1972). Anxiety: Current trends in theory and research (Vol. 1). New York, NY: Academic Press.

Spielberger, C. D., Gorsuch, R. L., Lushene, R., Vagg, P. R., \& Jacobs, G. A. (1983). Manual for the State-Trait Anxiety Inventory. Palo Alto, CA: Consulting Psychologists Press.

Tabachnick, B. G., \& Fidell, L. S. (2007). Using multivariate statistics (5th ed.). Pearson Education International.

Watson, D., Clark, L. A., \& Tellegen, A. (1988). Development and validation of brief measures of positive and negative affect: The PANAS scales. Journal of Personality and Social Psychology, 54, 1063-1070. https://doi.org/10.1037/0022-3514.54.6.1063 